\title{
Optimal supervisory control of steam generators operating in parallel
}

\author{
Vicente Costanza ${ }^{*}$ and Pablo S. Rivadeneira \\ Process Control Area, INTEC (UNL-CONICET), Santa Fe, Argentina
}

September 3, 2015

\begin{abstract}
A supervisory control working as a 'dynamic feedback' is substantiated for optimally allocating demands to a group of $n$ boilers in parallel. The set-points to each conventional controlled boiler are continuously changed while: (i) minimizing a combined cost, which is cumulative in time and takes into account the dynamics of all individual boilers, and (ii) generating a strategy that can cope with general disturbances, like changes in fuel composition and noisy measurements, i.e. with differences between the predicted and the measured values of the variables. The structure of the problem results in a $2 \mathrm{n}$ affine-linear model subject to a quadratic cost, and the resulting optimal control is also affine-linear with time-dependent coefficients, which do not depend on the total vapor demand. The methods are tested with a two case studies for 2 and 3 boilers. It is shown that this dynamical supervisory control leads to savings of at least $10 \%$ relative to nontrivial piecewise-constant strategies.
\end{abstract}

Keywords: supervisory control, optimal control, multilayer boilers, closedloop identification, boiler control

\section{Introduction}

Most of the heating systems, although not all, employ boilers to produce hot water or steam. All of the major industrial energy users devote significant proportions of their fossil fuel consumption to steam production: food processing (57\%), pulp and paper (81\%), chemicals (42\%), petroleum refining (23\%), and primary metals (10\%) [1]. Since industrial systems are very diverse, but often have major steam systems in common, boilers make a useful target for energy efficiency optimization. Also, heating systems in urban buildings consume a

\footnotetext{
${ }^{*}$ Corresponding author
} 
substantial proportion of their energy, and are responsible for about $25 \%$ of their total carbon emissions, as it is assessed by different surveys in Europe $[2,3]$. Despite the enormous effort made over the last decades to improve the energy efficiency of these heating systems, a huge potential for further energy saving still persists.

A boiler unit that produces steam is a critical component of the power plant system. One of the main concerns in recent years about the operation of a boiler unit has been the improving its controls as shown by the survey and consecutive experiments carried out in [2]. However, in the current literature and in the industrial environment, many control strategies have been applied to control the boiler as a process unit, ranging from standard methods like proportional integral derivative (PID) control [4] to intelligent and sophisticated methodologies as optimal control, sliding mode control, model predictive control and others, see $[4,6,5]$ and their references.

Other main concerns of plant operation have been the basic start-up strategy of steam boilers [7], and the minimization of $\mathrm{CO}_{2}$ emissions [8], where advanced techniques of optimization and adaptive monitoring schemes have been used to improve these objectives. However, the operation of groups of several boilers working in parallel has received little attention in recent technical literature.

In [9], an attempt to optimize energy losses to the environment (or equivalently to maximize the efficiency of the set of boilers), defined from theoretical relations among the many physical variables involved through a supervisory scheme control, is carried out in detail. That supervisory control assigns to each boiler its corresponding vapor production set-point by solving a static optimization problem by linear or nonlinear programming, which is difficult to adapt simultaneously to varying demands of steam generation in a real time operation.

Optimal allocation problems or supervisory control have a long tradition in engineering practice. In chemical processes, dynamic optimization frequently deals with distributing global energy demands of the plant into individual demands required by each member of a group of service equipment (boilers, heatexchangers, pumps, and the like), while minimizing some predetermined generalized cost. Usually these individual demands translate into set-points communicated to controllers of the PID type, which commonly are well tuned and perform efficiently. In this paper two aspects of this routine will be revised: (i) the methodology for deciding the individual set-points after a new total load is required from a group of boilers, and (ii) the convenience of changing these orders continuously in time, by optimizing some combined cost (typically the compromise vapor versus fuel) accumulated during a fixed finite horizon.

According to [10]: '... Real Time Optimizers have been implemented in order to optimize the cost of operating one or more loads. These Real Time Optimizers have detected a steady-state load requirement and then have provided control signals that optimize the cost of operating the loads based on this steady state load requirement. In order to operate in this fashion, the Real Time Optimizers have had to wait for transient process disturbances to settle out so that a steady state condition exists before such Optimizers can invoke their op- 
timization procedures. However, for processes with slow dynamics and/or high levels of disturbances, the dependence of Real Time Optimizers on steady-state information substantially deteriorates the performance of the control system, as no optimization is performed during the transients created by disturbances such as changes in set-point and/or changes in load.'

Other standard treatments follow static optimization lines, common to research operation engineering (see for instance [11], [12]). One of the patents related to the topic of this paper ([13], Fig. 1) asserts: 'Boiler optimization is included in on-line control of parallel boilers by multiplying the total heat per unit time which must be supplied to all parallel boilers by the percentage of the total heat which should be supplied to each boiler in order to substantially maximize energy efficiency. The result of such multiplication is the heat per unit time which should be supplied to each boiler.' Despite the 'on-line' qualification used in this description, it is clear that the 'heat per unit time' works as another set-point, and that this target is kept fixed while the total demand is constant. In other words, the resulting demand will be piecewise-constant, each time waiting for another static optimization routine to determine the new appropriate heat rate.

To the authors' knowledge, a thoroughly dynamic point-of-view has only been applied to particular situations, like redundant control and related problems [14]. Here an original 'dynamic feedback' strategy will be sought, in the sense that the set-points to each boiler will be allowed to be continuously changed while: (i) minimizing a combined cost, which is cumulative in time and takes into account the dynamics of all the individual boilers, and (ii) generating an optimal control that can cope with general disturbances, like changes in fuel composition, noisy measurements, etc., i.e. with differences between the predicted and the measured values of the variables. With these objectives in mind, a dynamics for the responses of each boiler to a new set-point indication will be assessed, directly from experimental data. Then the whole group of $n$ boilers will be assembled into a general model with an $(n-1)$-dimensional control vector associated with heat demands, the remaining one determined by the residue with respect to the global demand, which is known during each optimization time horizon. This new 'big' system, together with a typical quadratic cost functional, conform an optimal control problem that has a nice mathematical solution, namely an affine-linear feedback law with time-variant coefficients. Both the proportional coefficient and the feed-through term in the control law can be calculated only once for a unitary global demand, and stored in memory, the updating for another demand being straightforward. The nature of the modeling also admits a stochastically optimal handling of noisy and systemic perturbations, and eventually a suboptimal online correction [15] of the feedback law due to hard restrictions on control values [9]. In summary, previous papers mostly want to improve the servo control of just a single boiler in different environments, or to allocate steady targets to several boilers. In the first case, they attempt to increase the speed in reaching the set-points provided by the supervisory control. In the second one, they assume that the values of the set-points will be kept constant during a certain period of time (as far as 


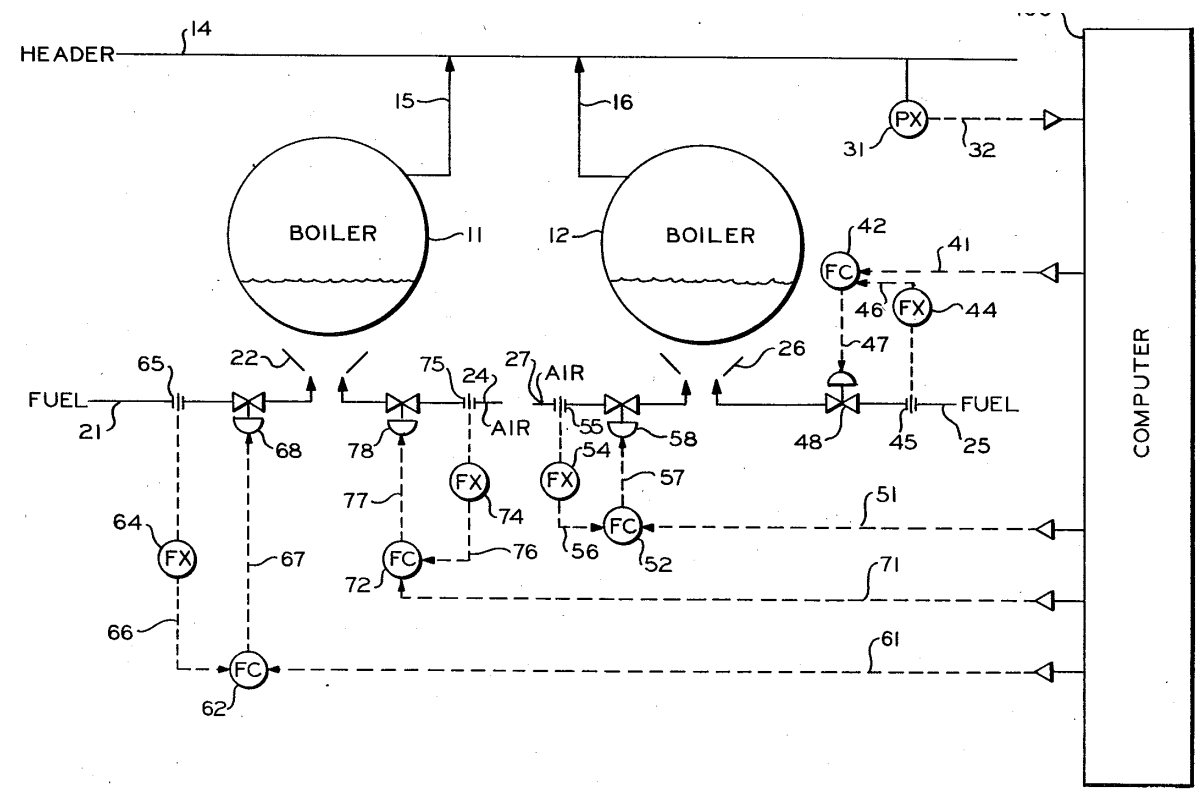

Figure 1: Scheme showing the control instrumentation for two boilers in parallel, feeding a unique header. Details are given in [13].

the total vapor demand does not change). The main contribution of our work, by allowing the set-points to be time-varying for all members of the group, even when the total vapor demand remains constant, is then to find their optimal evolution with respect to a quadratic cost criterion.

The rest of the article will be organized as follows: in Section 2 the theoretical setup of the problem is posed and the methods to solve it are developed, i.e. the modeling for the dynamics and the design of the cost objective are made explicit, the deterministic optimal solution analytically found, the procedure for updating the feedback under changes in the global demand is justified, and stochastic aspects are substantiated. Section 3 is devoted to numerical calculations and validations, and all issues illustrated for the case studies associated with groups of 2 and 3 boilers. The last Section exposes the conclusions.

\section{Theoretical setup}

\subsection{State space models: modeling and identification.}

In what follows it will be assumed that a group of boilers or similar service equipment is in operation as a part of an industrial plant, its members working in parallel, evolving within the admissible range of their main variables, and that each member is efficiently controlled according to conventional engineering 
practice. What is treated here is how to continuously change the set-point to each member of the equipment in order to optimize the global economic cost.

In Figure 3, registered data for the variables of interest associated to one boiler in real operation are shown. It is observed that the vapor production attempts to meet the demand. The demand functions as a target communicated by some supervisory command, and it is kept constant (typically as a fixed portion of the vapor required from the whole set of boilers) during a period of time until a new demand is decided. Since the ability to follow the individual demand is apparently accurate and fast, other questions arise, for instance: (i) are the piecewise-constant assignments to each member of the group optimally decided in some sense?, (ii) would it be possible to dynamically change the individual demands to improve the performance of the whole group?.

It is clear that trying to answer any of the previous questions should not involve modifying the control instrumentation already attached to each boiler, which usually involves finely tuned and long-tested PID controllers. Rather, the system of study should initially be a well controlled boiler, whose new input will be a time-varying vapor demand. The vapor actually produced should be a part of the state, since this will be the variable required to evolve in response to the input. In addition, vapor production implies fuel consumption, so the simplest description of the dynamics should involve an interaction between these two variables, chosen to determine the state of the system in a first attempt.

After applying several identification models (see Figure 3) to a group of real boilers meeting the conditions above, it was observed that the behavior of each controlled steam generator was well enough described by linear systems. In the general case, $n$ boilers in parallel are optimized, whose identified linear models result in

$$
\dot{x}_{i}=A_{i} x_{i}+B_{i} u_{i}, y_{i}=x_{i}, i=1, \ldots n ;
$$

where $x_{i}:=\left(x_{i 1}, x_{i 2}\right)^{\prime}$ is the state vector for boiler $i, x_{i 1}$ : vapor produced, $x_{i 2}$ : fuel consumed, $u_{i}$ : vapor demanded, all expressed in energy units, and $y_{i}$ : the output, equivalent to the state. For each boiler the matrices $A_{i}, B_{i}$ are $2 \times 2$ and $2 \times 1$, respectively.

When a total demand $\alpha$ is required to be supplied by the $n$ boilers, then necessarily

$$
u_{n}=\alpha-u_{1}-\cdots-u_{n-1} .
$$

Therefore, despite the facts that $n$ set-points are to be ordered to the $n$ boilers, there exist only $n-1$ degrees of freedom for treating the whole set. This leads to a possible setup for the optimal allocation problem to $n$ units working in parallel. After redefining

$$
x:=\left(x_{1}^{\prime} \vdots x_{2}^{\prime} \vdots \ldots \vdots x_{n}^{\prime}\right)^{\prime} ; u:=\left(u_{1}, \cdots, u_{n-1}\right)^{\prime} ;
$$

the dynamics for the set of boilers, under the restriction implied by Equation (1), becomes a $2 n$-dimensional linear-affine system with an $(n-1)$-dimensional control variable, namely

$$
\dot{x}=\hat{A} x+\hat{B} u+\varphi .
$$


with coefficient matrices

$$
\hat{A}:=\left(\begin{array}{cccc}
A_{1} & \cdots & 0 & 0 \\
\vdots & \ddots & \vdots & \vdots \\
0 & \cdots & A_{n-1} & 0 \\
0 & \cdots & 0 & A_{n}
\end{array}\right), \hat{B}:=\left(\begin{array}{ccc}
B_{1} & \cdots & 0 \\
\vdots & \ddots & \vdots \\
0 & \cdots & B_{n-1} \\
-B_{n} & \cdots & -B_{n}
\end{array}\right), \varphi:=\alpha\left(\begin{array}{c}
0 \\
\vdots \\
0 \\
B_{n}
\end{array}\right) \text {, }
$$

Assuming that each model in Equation (1) is in canonical form (controllable and observable), it follows from the form of matrix $\hat{A}$ that the global system

will be uniformly bounded-input bounded-output (UBIBO) if and only if the same is true for each individual subsystem (boiler). Therefore, under these assumptions, since the UBIBO property is equivalent to internal stability for each boiler, the global system will be externally stable when each identified matrix $A_{i}$ is stable (its eigenvalues have negative real parts). It can be concluded then that the stability properties of the $2 n$-dimensional system will not differ from those already present in the models of each one of the controlled boilers.

\subsection{Supervisory control: an optimal deterministic feed- back.}

The optimal control problem motivated in the previous section will be posed for a quadratic objective cost functional of the form

$$
\mathcal{J}(u)=\int_{0}^{T}\left[(x(t)-\bar{x})^{\prime} Q(x(t)-\bar{x})+u^{\prime}(t) R u(t)\right] d t+(x(T)-\bar{x})^{\prime} S(x(T)-\bar{x}),
$$

restricted to the affine system of Equations (4-5). The target

$$
\bar{x}:=\left(\bar{x}_{1}^{\prime} \vdots \bar{x}_{2}^{\prime} \vdots \ldots \vdots \bar{x}_{n}^{\prime}\right)^{\prime}
$$

is a design parameter vector, which will be assumed here to have the form

$$
\bar{x}_{i}=\left(\bar{x}_{i 1}, 0\right)^{\prime}, i=1, \ldots, n .
$$

As usual, $Q$ and $S$ will be nonnegative $2 n \times 2 n$ symmetric matrices, and $R$ will be $(n-1) \times(n-1)$ and positive definite. The coefficients of $Q, R, S$ will be decided according to characteristics of each application. It seems most probable that $Q$ and $S$ be constructed from $2 \times 2$ symmetric submatrices displayed in diagonal, one such a submatrix related to each boiler. In the same fashion, $R=\operatorname{diag}\left(r_{1}, \ldots, r_{n-1}\right)$, with all $r_{i}>0$, would be a practical choice for weighting the control energy effort associated to the problem. Notice that, in order to take into account that there exist $n$ boilers and only $n-1$ available coefficients $r_{i}$, a sensible compromise would be to adopt

$$
r_{i}=\tilde{r}_{i}+\frac{\tilde{r}_{n}}{n-1}, i=1, \ldots, n-1,
$$


where $\tilde{r}_{i}$ is the weight of the control corresponding to the demand assigned to the individual boiler $i$, for $i=1, \ldots n$. Formula 9 satisfies

$$
\sum_{i=1}^{n-1} r_{i} u_{i}^{2}=\sum_{i=1}^{n} \tilde{r}_{i} u_{i}^{2}
$$

when $u_{i} \equiv \alpha / n$ (a typical choice for $\bar{x}_{i 1}$ ), $i=1, \ldots n$, and it is a good approximation under the following restrictions: (i) $u_{i} \in[0, \alpha]$, and (ii) $u_{1}+\ldots+u_{n}=\alpha$, as it is the case here. As expected, this choice of $r_{i}$ verifies: $\lim _{n \rightarrow \infty} r_{i}=\tilde{r}_{i}$, for $i=1, \ldots n$.

The affine linear-quadratic retains some features of the classical LQR problem. For instance, its Hamiltonian $H$ [17]

$$
H(x, \lambda, u):=L+\lambda^{\prime} f=(x-\bar{x})^{\prime} Q(x-\bar{x})+u^{\prime} R u+\lambda^{\prime}(\hat{A} x+\hat{B} u+\varphi)
$$

is minimized by the same expression as in the LQR case:

$$
u^{0}(x, \lambda)=-\frac{1}{2} R^{-1} \hat{B}^{\prime} \lambda
$$

but the $u$-minimal Hamiltonian $H^{0}$ has an extra term $\lambda^{\prime} \varphi$, precisely,

$$
H^{0}(x, \lambda):=H\left(x, \lambda, u^{0}(x, \lambda)\right)=(x-\bar{x})^{\prime} Q(x-\bar{x})-\frac{1}{4} \lambda^{\prime} \hat{W} \lambda+\lambda^{\prime}(\hat{A} x+\varphi),
$$

where the usual notation $\hat{W}:=\hat{B} R^{-1} \hat{B}^{\prime}$ has been introduced. The affine term suggests that a complete quadratic form might be proposed for a value function $V[16]$, for instance

$$
V(t, x):=x^{\prime} P(t) x+2 \xi^{\prime}(t) x+\sigma(t),
$$

formally for some time-varying (in principle symmetric) $2 n \times 2 n$ matrix $P$, a $2 n$ column vector $\xi$, and a scalar factor $\sigma$.

The Hamilton-Jacobi-Bellman (HJB) equation

$$
\frac{\partial V}{\partial t}(t, x)=-H^{0}\left(x,\left(\frac{\partial V}{\partial x}\right)^{\prime}(t, x)\right)
$$

and its final condition

$$
V(T, x)=(x(T)-\bar{x})^{\prime} S(x(T)-\bar{x})
$$

must be satisfied by the proposed value function and its partial derivatives

$$
\begin{aligned}
\frac{\partial V}{\partial t}(t, x) & =x^{\prime} \dot{P}(t) x+2 x^{\prime} \dot{\xi}(t)+\dot{\sigma}(t), \\
\left(\frac{\partial V}{\partial x}\right)^{\prime}(x(t)) & =2[P(t) x+\xi(t)]
\end{aligned}
$$


Since these equalities involve second order polynomials in $x$, their coefficients must also be equal, which result in the following system of ODE's:

$$
\begin{aligned}
\dot{P} & =P \hat{W} P-\hat{A}^{\prime} P-P \hat{A}-Q, \quad P(T)=S, \\
\dot{\xi} & =-(\hat{A}-\hat{W} P)^{\prime} \xi-P \varphi+Q \bar{x}, \quad \xi(T)=-S \bar{x}, \\
\dot{\sigma} & =\xi^{\prime} \hat{W} \xi-\bar{x}^{\prime} Q \bar{x}-2 \varphi^{\prime} \xi, \quad \sigma(T)=\bar{x}^{\prime} S \bar{x} .
\end{aligned}
$$

Their solutions allow to express the optimal control $u^{*}$ in the form

$$
u^{*}(t)=u^{0}\left(x^{*}(t),\left(\frac{\partial V}{\partial x}\right)^{\prime}\left(t, x^{*}(t)\right)\right)=-R^{-1} \hat{B}^{\prime}\left[P(t) x^{*}(t)+\xi(t)\right],
$$

where $x^{*}$ denotes the optimal state, and also to calculate the optimal cost from

$$
V\left(0, x_{0}\right)=x_{0}^{\prime} P(0) x_{0}+2 x_{0}^{\prime} \xi(0)+\sigma(0) .
$$

Equation (19) coincides with the Riccati Differential Equation for the LQR problem with coefficients $(\hat{A}, \hat{B}, Q, R, S)$, and turns to be uncoupled from the remaining two ODEs. This finding is consistent with the behavior of the corresponding solutions of equations $(20,21)$, since for such an LQR problem: (i) $\varphi=\bar{x}=0$, implying $\xi(\cdot) \equiv 0$ and $u^{*}(t)=-R^{-1} \hat{B}^{\prime} P(t) x(t)$; and in turn (ii) $\sigma(\cdot) \equiv 0$ and $V\left(0, x_{0}\right)=x_{0}^{\prime} P(0) x_{0}$. It should be noted that, even when the problem at hand does not possess the LQR structure, still the equation (22) can be interpreted as a linear-affine feedback law $u_{f}$, precisely

$$
u_{f}(t, x):=-R^{-1} \hat{B}^{\prime}[P(t) x+\xi(t)],
$$

and from Bellman's Principle it could be asserted that, if at time $t$ the actual state $x$ differs from the expected optimal state $x^{*}(t)$, still the optimal control at that time, denoted $u_{x}^{*}(t)$, can be computed as $u_{f}(t, x)$. This property makes the previous results robust against sporadic state errors.

\subsection{Handling changes in the total demand $\alpha$. Fixed versus receding horizon strategies.}

Equations $(19,20)$ have final (instead of initial) conditions and therefore can not be numerically integrated online with the process. They need to be solved offline and stored in the memory of the controller. This is an inconvenience common to the LQR, servo, and tracking problems, for which the feed-through terms and similar objects must be updated for the whole time-horizon in case the reference signal is modified. Fortunately, in the present case the calculation of the time-varying coefficient $\xi(\cdot)$ of the feedback law is required to be computed only once, namely for a unitary total demand $(\alpha=1)$, and the same thing applies to the cost coefficient $\sigma(\cdot)$. These assertions are conveyed in precise terms by the next two equations: 


$$
\begin{gathered}
u^{*}(t)=-R^{-1} \hat{B}^{\prime}[P(t) x(t)+\alpha \tilde{\xi}(t)], \\
J^{*}=V\left(0, x_{0}\right)=x_{0}^{\prime} P(0) x_{0}+2 \alpha x_{0}^{\prime} \tilde{\xi}(0)+\alpha^{2} \tilde{\sigma}(0),
\end{gathered}
$$

where $\tilde{\xi}, \tilde{\sigma}$ denote the coefficients calculated for $\alpha=1$, or equivalently for $\tilde{\varphi}:=$ $\varphi / \alpha=\left(0^{\prime} \vdots 0^{\prime} \vdots \ldots \vdots B_{n}^{\prime}\right)^{\prime}, \tilde{x}:=\bar{x} / \alpha=\left(\check{x}_{1}, 0, \check{x}_{2}, 0, \ldots, \check{x}_{n}, 0\right)^{\prime}, \sum_{i=1}^{n} \check{x}_{i}=1$. Equations $(25,26)$ can be justified as follows:

(i) the solution to equation (20) with final condition $\xi(T)=-S \bar{x}=-\alpha S \tilde{x}$ is

$$
\xi(t)=\Psi(t, T)\left\{-S \bar{x}+\int_{T}^{t} \Psi(t, \tau)[-P(\tau) \varphi+Q \bar{x}] d \tau\right\}=\alpha \tilde{\xi}(t),
$$

where $\Psi(t, T)$ is the fundamental matrix associated with the linear (time-varying) system

$$
\dot{z}=-(\hat{A}-\hat{W} P(t))^{\prime} z
$$

(ii) and similarly, for equation (21),

$$
\sigma(t)=\bar{x}^{\prime} S \bar{x}+\int_{T}^{t}\left[\xi^{\prime}(\tau) \hat{W} \xi(\tau)-\bar{x}^{\prime} Q \bar{x}-2 \varphi^{\prime} \xi(\tau)\right] d \tau=\alpha^{2} \tilde{\sigma}(t) .
$$

Now it should be decided how to handle changes in the total demand when they occur in some interior point $t$ of a period $\left[t_{0}, t_{0}+T\right]$. Let us assume that, in such a case, the optimization of the system is desired to be continued, at least for another interval of duration T. Some 'receding-horizon' decision has to be made. Would it be advisable: (i) to wait until the end of the first period before updating the control (25) with the new value of $\alpha$ ?, or rather: (ii) to update the control as soon as possible?. Other combinations are possible, for instance: (iii) to initiate another optimization period each time $\alpha$ changes. It is known that none of these strategies will be strictly optimal. Nevertheless, their performances were assessed, since in real operation the intervals of constant demand are not always of the same duration.

\subsection{Optimal filtering and stochastic control.}

Noisy perturbations in the system parameters of each boiler, and in the measurement and communication of signals, are known to occur during real process operation. The common set up for these influences over the deterministic models assumed in previous sections for boilers $i=1, \ldots, n$ is the following

$$
\begin{gathered}
\dot{x}_{i}(t)=A_{i} x_{i}(t)+B_{i} \tilde{u}_{i}(t)+r_{1 i}, \\
y_{i}=C_{i} x_{i}+r_{2 i},
\end{gathered}
$$


where the notation $\dot{x}_{i}$ should be understood as the differential of a Brownian process associated with the state of boiler $i$, resulting from the existence of zeromean white noise $r_{1 i}$ fluctuations on the environment conditions; $\tilde{u}_{i}$ denoting the $i^{t h}$-component of the input variable for $i=1, \ldots, n-1, \tilde{u}_{n}:=\alpha-\sum_{i=1}^{n-1} \tilde{u}_{i}$; and where $r_{2 i}$ are the zero-mean white noises in the measurements of the outputs $y_{i}$, which in this case are conceptually the same thing as the states, i.e. it is assumed that each subsystem $i$ is observable (which is clearly true in most modern plants, with $C_{i}=I_{2}$, the identity matrix of dimension 2).

In this context, the optimal filtering problem for each boiler is known to be solvable $[18,19]$ through the following pair of equations (for $i=1, \ldots, n$ ):

$$
\begin{gathered}
\dot{\hat{x}}_{i}(t)=A_{i} \hat{x}_{i}(t)+B_{i} \tilde{u}_{i}(t)+L_{i}\left(y_{i}(t)-C_{i} \hat{x}_{i}(t)\right) ; \hat{x}_{i}(0)=\mathbb{E}\left[x_{0}\right], \\
\dot{\Pi}_{i}=A_{i}^{\prime} \Pi_{i}+\Pi_{i} A_{i}+\hat{Q}_{i}-\Pi_{i} C_{i}^{\prime} \hat{R}_{i}^{-1} C_{i} \Pi_{i} ; \Pi_{i}(0)=\operatorname{Cov}\left(x_{0 i}\right),
\end{gathered}
$$

which can be integrated online with the process, and where $\hat{x}_{i}$ is the best estimation of the state $x_{i} ; r_{1 i}$ and $r_{2 i}$ are stochastic differentials of Brownian motions with covariance matrices $\hat{Q}_{i}$ and $\hat{R}_{i}$ respectively, with $\hat{R}_{i}$ invertible; $L_{i}:=$ $\Pi_{i} C_{i}^{\prime} \hat{R}_{i}^{-1}$ denotes the 'gain' of the filter, which works like an observer; and $\Pi_{i}$ is the dynamical covariance, solution to the Riccati-type ODE (31).

As a consequence of a straightforward extension of the Separation Principle $[20]$ to this linear-affine problem, the stochastic optimal control for the whole system, which minimizes the cost

$\mathcal{J}_{\text {sto }}(\tilde{u})=\mathbb{E}\left[\int_{0}^{T}\left\{\left(x_{\tilde{u}}(t)-\bar{x}\right)^{\prime} Q\left(x_{\tilde{u}}(t)-\bar{x}\right)+\tilde{u}^{\prime}(t) R \tilde{u}(t)\right\} d t+\left(x_{\tilde{u}}(T)-\bar{x}\right)^{\prime} S\left(x_{\tilde{u}}(T)-\bar{x}\right)\right]$,

with respect to $\tilde{u}$, results in

$$
\hat{u}^{*}(t)=-R^{-1} \hat{B}^{\prime}[P(t) \hat{x}(t)+\alpha \tilde{\xi}(t)],
$$

where the estimation of the whole state vector is used in the feedback law, i.e.

$$
\hat{x}:=\left(\hat{x}_{1}^{\prime}: \hat{x}_{2}^{\prime}: \ldots: \hat{x}_{n}^{\prime}\right)^{\prime} \text {. }
$$

A scheme describing the relations amongst the signals involved in these results is given in Figure 2.

\section{Case studies}

\subsection{Two boilers in parallel.}

Real numerical data for the state and control variables were extracted from records of instruments attached to a set of boilers serving an industrial process 


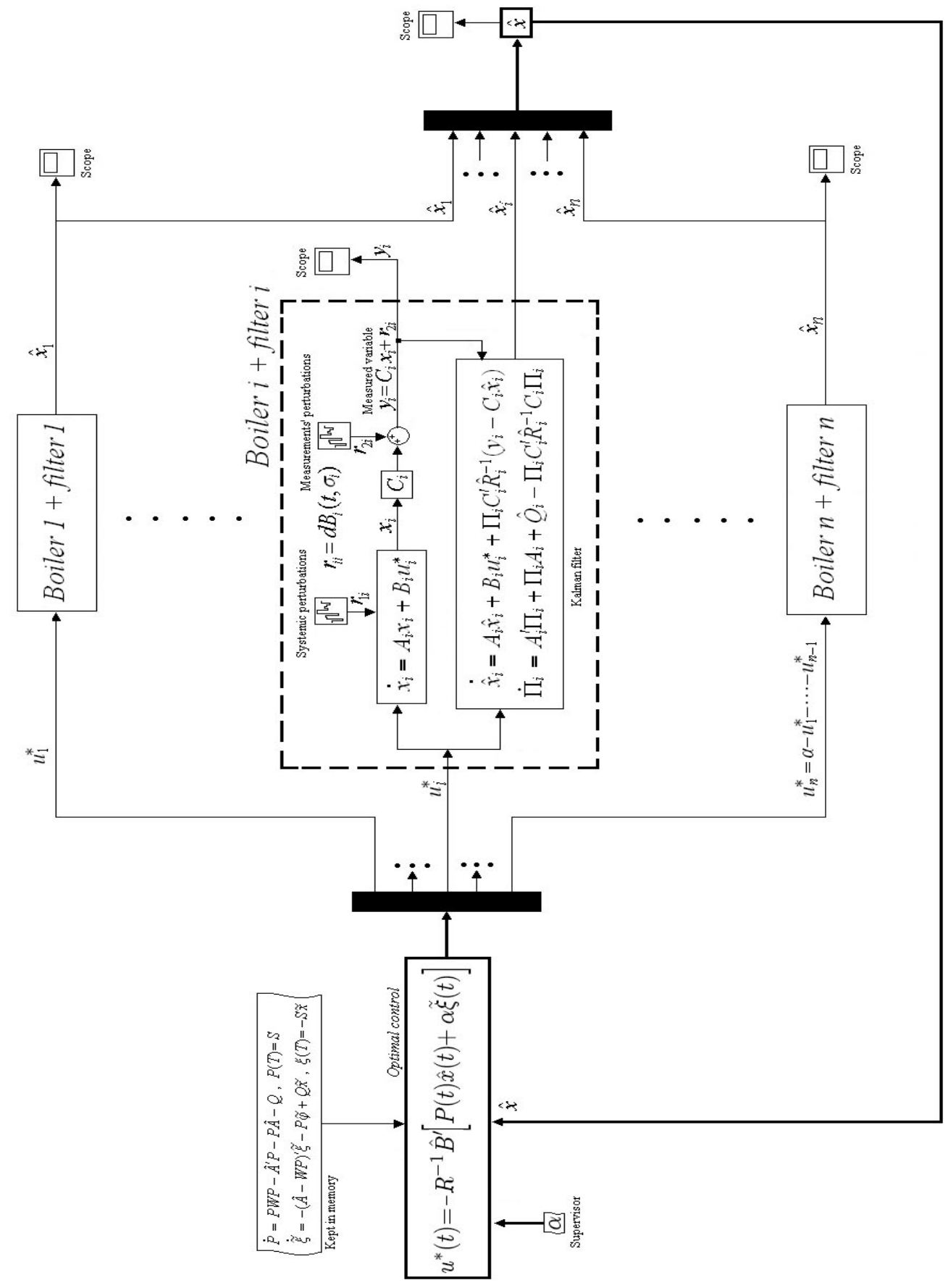

Figure 2: Flowsheet illustrating the signals involved in the filtering stage, and construction of the stochastic optimal control for all boilers. 


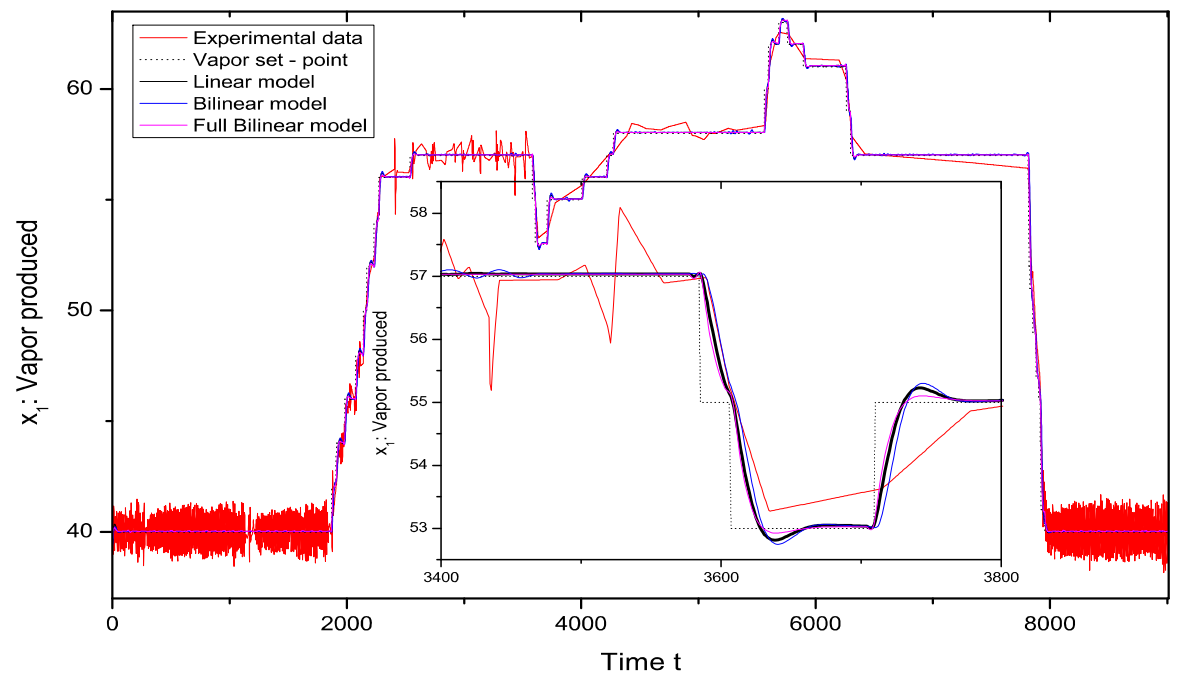

Figure 3: Main frame: real registered data corresponding to a controlled boiler along with the results of the identification model for the whole horizon. In zoom: different identification results for $x_{1}$, showing that the linear model is good enough to represent the process.

plant. The relevant variables were recorded every 10 seconds. For one of the boilers, a limited period of the available records are depicted in Figure 3. Each boiler is already controlled by a PID. A linear model and two different bilinear models were proposed to represent the dynamics of the controlled boilers. It was found that the linear model was good enough in the full range covered by the variables, so the simplest model was chosen in consideration to the existing theoretical support concerning linear control systems (Figure 3.)

Since the identified parameters cover a wide range of variation of state and control values, the model obtained should be regarded as a 'dynamical' version of each conventionally-controlled boiler. Actually, identified models are in principle more reliable than curves provided by the factory, since the dynamics is approximated from I/O data coming from really operating equipment, and they can be updated systematically if desired. The model also takes into account the whole controlling instrumentation attached to the boiler (see Figure 1), and the actual way it is working at the recording time (which not always remains as at the moment of installation). 
After linear identification of two boilers the resulting coefficients were

$$
\begin{aligned}
& A_{1}=\left(\begin{array}{cc}
-0.1016 & 0.0426 \\
-0.1936 & -0.0290
\end{array}\right), A_{2}=\left(\begin{array}{cc}
-0.2016 & 0.0426 \\
-0.1936 & -0.1290
\end{array}\right), \\
& B_{1}=\left(\begin{array}{l}
0.0434 \\
0.2333
\end{array}\right), B_{2}=\left(\begin{array}{l}
0.1341 \\
0.3543
\end{array}\right), C_{1}=C_{2}=\left(\begin{array}{ll}
1 & 0 \\
0 & 1
\end{array}\right) .
\end{aligned}
$$

The realizations are canonical, the eigenvalues of $A_{1}, A_{2}$ are $-0.0653 \pm 0.0832 i$ and $-0.1653 \pm 0.0832 i$, respectively, showing that the second boiler has a faster response than the first one. On the other hand, for each boiler, the stationary output $y_{\infty}$ corresponding to a constant input $u(\cdot) \equiv \kappa$ is $y^{\infty}=-A^{-1} B \kappa$. For the two boilers at hand,

$$
y_{1}^{\infty}=\left(\begin{array}{l}
1.0003 \\
1.3669
\end{array}\right) \kappa, y_{2}^{\infty}=\left(\begin{array}{c}
0.9457 \\
1.3273
\end{array}\right) \kappa .
$$

This shows that the faster (second) boiler will be subject to an off-set (first component of $y^{\infty}$ ) of around $5.5 \%$, much bigger than the offset of the first boiler. Therefore, the coefficients of each realization convey enough information about the performance expected from each PID-controlled boiler.

Deterministic optimal control: An interesting question concerning the optimal control design is how to choose the parameters $Q, R$ and $S$ involved in the cost function. The choosing of these matrices must lead to 'acceptable' levels of $x(t), u(t)$, and $x(T)$. A classical approach [21] initializes $Q_{i i}, R_{i i}$ and $S_{i i}$ tentatively, and then modify these values by trial and error, to reach a compromise among response time, damping and control effort. In more recent literature, there are several papers covering this subject, for instance [22, 23]. Here, just for illustration, simple diagonal weights were adopted, so that they indicate how much each state and input deviation contribute to the overall cost. After translating the amounts of vapor and fuel to economic units, and verifying that the compromise suggested in [21] was properly met, the following values were found appropriate

$$
\begin{aligned}
& Q=\left(\begin{array}{cccc}
q_{1} & 0 & 0 & 0 \\
0 & q_{2} & 0 & 0 \\
0 & 0 & q_{1} & 0 \\
0 & 0 & 0 & q_{2}
\end{array}\right), S=\left(\begin{array}{cccc}
s_{1} & 0 & 0 & 0 \\
0 & s_{2} & 0 & 0 \\
0 & 0 & s_{1} & 0 \\
0 & 0 & 0 & s_{2}
\end{array}\right), \bar{x}=\alpha\left(\begin{array}{c}
\tilde{x} \\
0 \\
\tilde{x} \\
0
\end{array}\right)(37) \\
& R=I_{n-1}, q_{1}=50, q_{2}=0.5, s_{1}=s_{2}=10, \tilde{x}=0.5, T=20, \alpha=150 .
\end{aligned}
$$

These were used in a simulation run: firstly $P, \xi$, $\sigma$, were calculated, and then introduced in the dynamics to obtain the optimal control $u^{*}$ shown in Figure 4. Early in the process the resulting control puts a high demand on the second (faster) boiler, but towards the end the first boiler is privileged due to its better accuracy (lower off-set). In between, the controls to both boilers oscillate around $\alpha / 2$ in a nontrivial pattern. The resulting states evolutions are illustrated in Figure 5. The optimal cost $J^{*}$ was compared against the 


\begin{tabular}{cccccccc}
\hline & $k=0.3$ & $k=0.35$ & $k=0.4$ & $k=0.5$ & $k=0.55$ & $k=0.6$ & $k=0.65$ \\
\hline$D_{k}$ & $47 \%$ & $23 \%$ & $15 \%$ & $9 \%$ & $25 \%$ & $40 \%$ & $70 \%$ \\
\hline
\end{tabular}

Table 1: Optimal cost compared against the outcome of applying a constant setup of different magnitude, where $k$ defines the magnitude of the control applied to the first boiler, and $D$ shows the porcentage of cost savings giving by Eq. (38).

outcome of applying different constant set-points of magnitude $u_{1} \equiv k \alpha, u_{2} \equiv$ $(1-k) \alpha$, and by calculating the corresponding costs $J_{k}$ arising from equation (6). Relative cost savings

$$
D_{k}:=100 \frac{J_{k}-J^{*}}{J^{*}},
$$

are reported in Table 1.

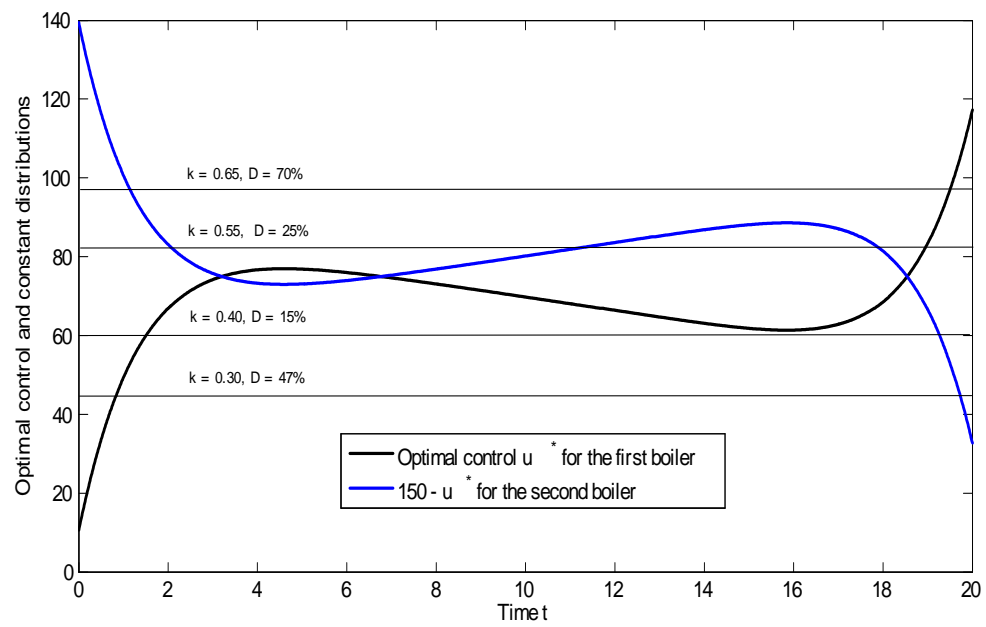

Figure 4: Optimal control for two boilers in parallel. Horizontal lines describe different constant controls as in Table 1.

Receding horizon strategies: As it was announced in the theoretical setup section, there are situations in the operation of a group of the boilers when the total demand (or set-point for the whole group) is changed (see Figure 3). This change may occur even in the middle of the optimization period established before. To illustrate these situations consider the following stage: for two boilers that receive at $t=0$ a total vapor demand $\alpha=150$, to be met in a time span $T=20$, but at time $t=10$ the total demand is changed to $\alpha=250$ by the 


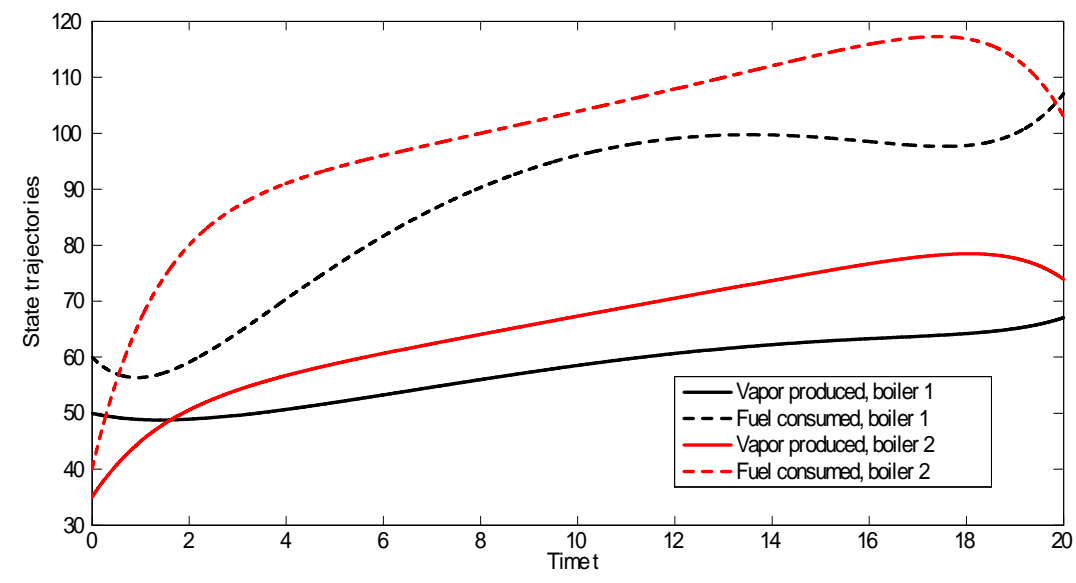

Figure 5: States resulting from the optimal control applied to two boilers in parallel.

supervisor system. It is assumed that $P(t), \tilde{\xi}(t)$ are stored for $t \in[0,20]$, that they will not be recalculated for a different horizon, and that the operation will be kept during two equal intervals, each of duration $T=20$.

The criterion for comparing the three suboptimal strategies was

$$
\mathcal{J}_{o p}=\int_{0}^{40}(x(t)-\bar{x})^{\prime} Q(x(t)-\bar{x}) d t+(x(40)-\bar{x})^{\prime} S(x(40)-\bar{x}),
$$

where $\bar{x}=\alpha / 2$. Here the cost associated with controlling the system was neglected, emphasizing the purely technical efficiency. The different options under evaluation for two boilers in parallel were:

- Strategy 1. At the beginning, $\alpha=150$ and the optimal control was calculated from equation (25), with coefficients $P(t), \tilde{\xi}(t)$ corresponding to $T=20$. At $t_{1}=10$ a total vapor demand $\alpha=250$ was ordered by the plant, but the calculation of the control was kept unaffected until the first period ended at $t=20$. Then, a second period was started with the same $P(t), \tilde{\xi}(t), T=20$ ( $t$ here is the real time minus 20 ), but now with $\alpha=250$.

- Strategy 2. Same as in case 1 for $t \in[0,10]$. At $t=10$ a new $\alpha=250$ is ordered. Now, a new period with $T=20$ and $\alpha=250$ is initiated, and the control is applied with the $P(t), \tilde{\xi}(t)$ stored in memory, but $t$ here is the real time minus 10. For $t \in[30,40]$ the first half of a new period with $T=20$ and $\alpha=250$ is executed. 


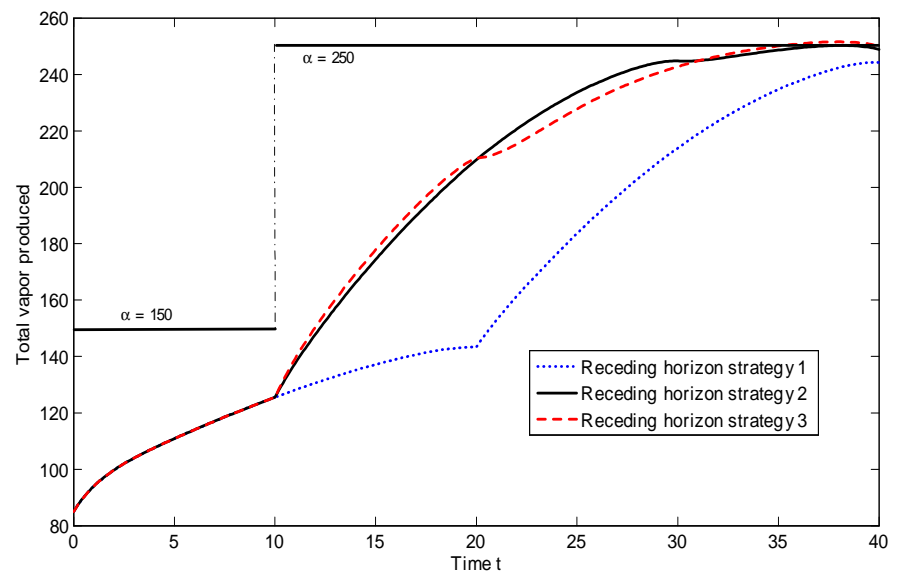

Figure 6: Total vapor produced in the three receding horizon strategies

- Strategy 3. Same as in case 2 for $t \in[0,10]$, until $\alpha=250$ is ordered. In this case only the value of $\alpha$ is changed in equation (25) for the control, but the real time coincides with $t$ for $t \in[10,20]$. Then, a second period was started with the same $P(t), \tilde{\xi}(t), T=20$ ( $t$ here is the real time minus $20)$, but now with $\alpha=250$.

In the calculation of the cost criterion of equation (39), the vapor targets were the constants $\bar{x}_{1}=150 / 2$ for $t \in[0,10]$, and $\bar{x}_{1}=250 / 2$ for $t \in$ $[10,40]$, in all strategies. The total value of $\mathcal{J}_{\text {op }}$ obtained for each strategy along the trajectory, denoted $\mathcal{J}_{o p}^{(i)}$, were $\mathcal{J}_{o p}^{(1)}=5.94 \times 10^{6}, \mathcal{J}_{o p}^{(2)}=$ $3.36 \times 10^{6}, \mathcal{J}_{o p}^{(3)}=3.40 \times 10^{6}$. The values of the final penalization were $6.68 \times 10^{5}, 6.12 \times 10^{5}$, and $6.18 \times 10^{5}$. The evolution of the control variable $u_{1}$ and the cost criterion are shown in Figure 7 . Cost considerations indicate that the change in the total demand (target) should be introduced as soon as possible in the calculation of the control, which discards strategy 1. The quantitative differences between strategies 2 and 3 are small, therefore strategy 3 should be preferred for its simpler implementation. The three strategies are illustrated in Figures 6 and 7 .

Stochastic control: Simulations for the two-boilers case are illustrated in Figures 8 , and 9 . The measurement noise was simulated with zero-mean and the covariance of the data in Figure 3, namely

$$
\hat{R}_{1}=\hat{R}_{2}=\left(\begin{array}{ll}
1.2349 & 0.0585 \\
0.0585 & 1.5770
\end{array}\right) \text {. }
$$

The systemic noise was neglected in this case, i.e. $\hat{Q}_{1}=\hat{Q}_{2}=0$. The 


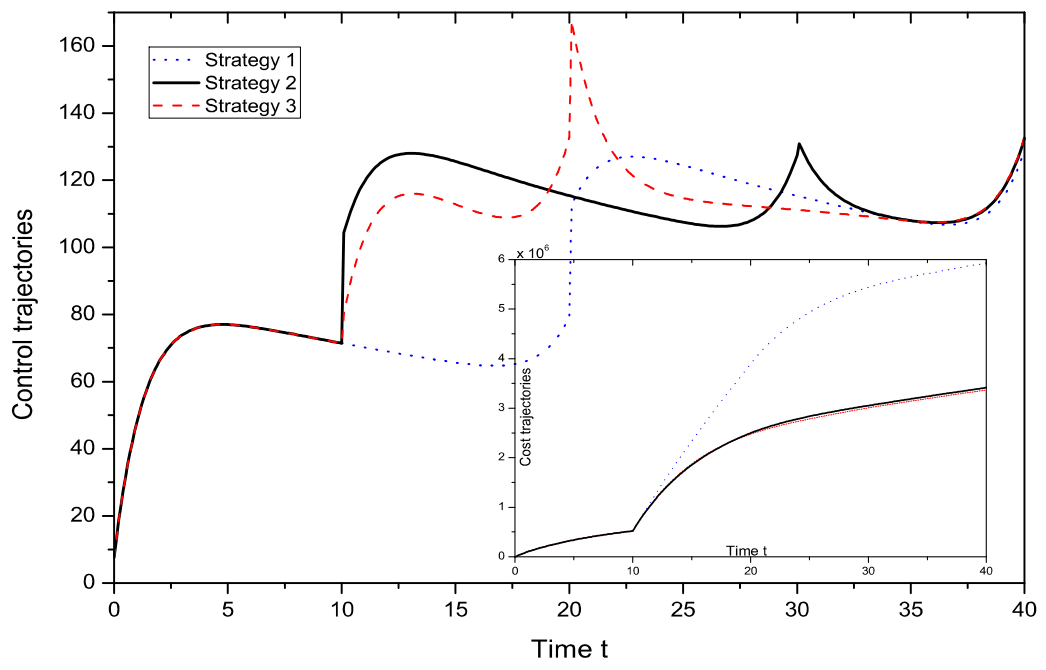

Figure 7: Main frame: Control variable in the three receding horizon strategies. Small frame: Cumulative evolution of the cost for the three receding horizon strategies.

deterministic coefficients used in the simulations were $q_{1}=50, q_{2}=0.5, R=$ $1.2, s_{1}=s_{2}=5, \tilde{x}=0.5, T=20, \alpha=150$.

A numerical partial confirmation of the stochastic optimality of the feedback law in Equation (33) was obtained by evaluating the costs corresponding to appropriate combinations of the trajectories in Figure 9; in precise terms,

$$
\begin{aligned}
& J_{1}:=\int_{0}^{T}\left[(\hat{x}(t)-\bar{x})^{\prime} Q(\hat{x}(t)-\bar{x})+\hat{u}^{* \prime}(t) R \hat{u}^{*}(t)\right] d t+(\hat{x}(T)-\bar{x})^{\prime} S(\hat{x}(T)-\bar{x}) \\
& J_{2}:=\int_{0}^{T}\left[(\check{x}(t)-\bar{x})^{\prime} Q(\check{x}(t)-\bar{x})+\check{u}^{\prime}(t) R \check{u}(t)\right] d t+(\check{x}(T)-\bar{x})^{\prime} S(\check{x}(T)-\bar{x})
\end{aligned}
$$

where the optimal stochastic feedback as in equation (24) is assessed in the cost $J_{1}$; and in the expression of $J_{2}$,

$$
\check{u}(t):=-R^{-1} \hat{B}^{\prime}[P(t) x(t)+\alpha \tilde{\xi}(t)],
$$

where $x(\cdot)$ was a numerical noisy zero-mean perturbation of the optimal $\hat{x}(\cdot)$, and $\check{x}(\cdot)$ denotes the numerical (deterministic) solution of the state equation (28) for inputs $\check{u}_{i}(\cdot), r_{1 i} \equiv 0, i=1, \ldots, n$. The resulting values were:

$$
\mathcal{J}_{\text {sto }}\left(\hat{u}^{*}\right) \cong J_{1}=834313<842986=J_{2} \cong \mathcal{J}_{\text {sto }}(\check{u}) \text {. }
$$




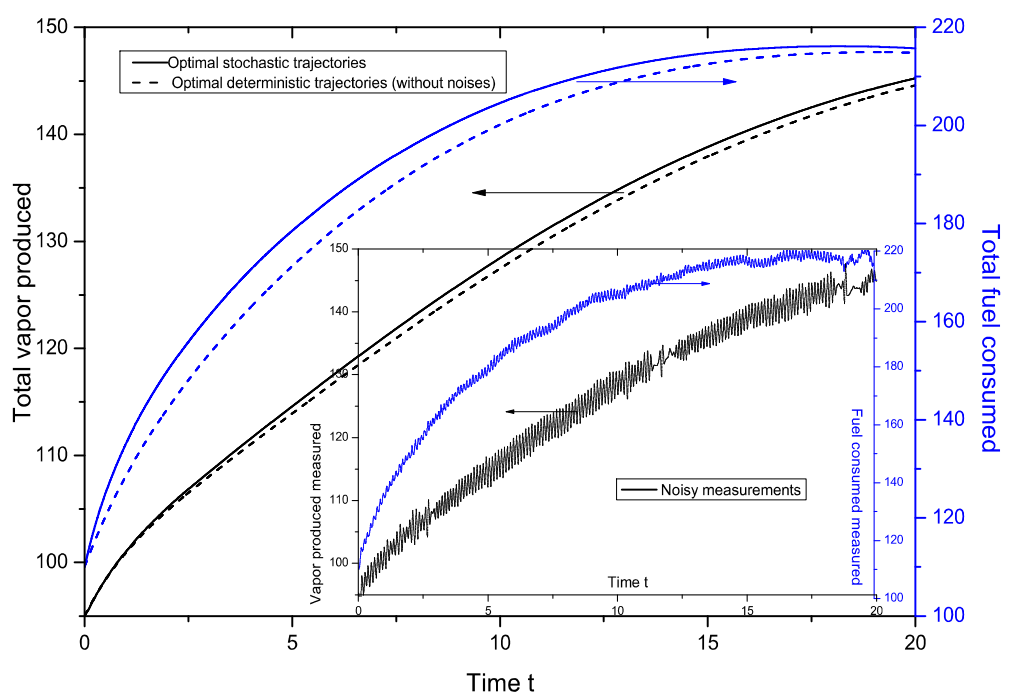

Figure 8: Main Frame: Optimal total vapor production and total fuel consumption for: (i) the deterministic problem and (ii) the stochastic problem with same coefficients. Small frame: Output variables when measurement perturbations of the order of the real data in Figure 3 are present.

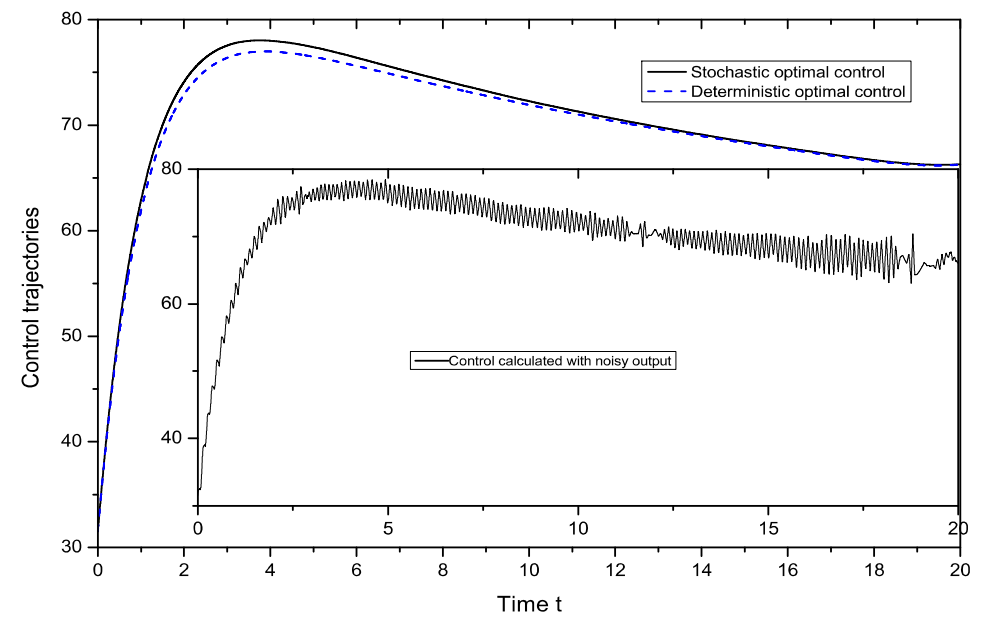

Figure 9: Main frame: optimal controls (first of two boilers) for the deterministic and stochastic problems with same coefficients. Small frame: control feedback calculated from optimal deterministic coefficients $P(),. \xi($.$) and noisy outputs$ with a covariance as in Equation (40). 


$$
\hat{u}^{*}(t)=-R^{-1} \hat{B}^{\prime}[P(t) \hat{x}(t)+\alpha \tilde{\xi}(t)],
$$

where the estimation of the whole state vector is used in the feedback law, i.e.

$$
\hat{x}:=\left(\hat{x}_{1}^{\prime} \vdots \hat{x}_{2}^{\prime} \vdots \cdots \vdots \hat{x}_{n}^{\prime}\right)^{\prime} \text {. }
$$

\subsection{Three boilers in parallel.}

It seems necessary to check the performance of the proposal for a larger group of boilers. Let us stay in the deterministic context for simplicity. By adding another set of data extracted from the same real plant alluded before, the matrices corresponding to identified linear models of three boilers in parallel, studied next, are:

$$
\begin{aligned}
& A_{1}=\left(\begin{array}{cc}
-0.1016 & 0.0426 \\
-0.1936 & -0.0290
\end{array}\right), B_{1}=\left(\begin{array}{l}
0.0434 \\
0.2333
\end{array}\right), \\
& A_{2}=\left(\begin{array}{cc}
-0.2016 & 0.0426 \\
-0.1936 & -0.1290
\end{array}\right), B_{2}=\left(\begin{array}{l}
0.1341 \\
0.3543
\end{array}\right), \\
& A_{3}=\left(\begin{array}{cc}
-0.0716 & 0.0426 \\
-0.1936 & -0.0010
\end{array}\right), B_{3}=\left(\begin{array}{l}
.01115 \\
0.1817
\end{array}\right) .
\end{aligned}
$$

In the simulations concerning these three boilers the total vapor demand was the same as before, $\alpha=150$, but the time horizon was now chosen near the setting time of the slowest boiler: $T=40$.

Quadratic cost. Just for illustrative purposes, the initial conditions for the three boilers were assumed identical for the three members, $x_{0}=(40,55,40,55$, $40,55)^{\prime}$, and the remaining parameters for the cost were kept in concordance to the case of two boilers.

The resulting optimal control for this case is depicted in Figure 10. Since the third boiler has the slowest response, it is observed that its participation increases near the end of the horizon as expected, but the control for all three boilers are qualitatively different from those in Figure 4.

The optimal cost was compared against the outcomes of applying constant set-points of different magnitude as follows:

$$
u_{k_{1} k_{2}}:=\left(u_{1}, u_{2}, u_{3}\right) ; u_{1} \equiv k_{1} \alpha, u_{2} \equiv k_{2} \alpha, u_{3} \equiv k_{3} \alpha=\left(1-k_{1}-k_{2}\right) \alpha,
$$

where the weight $k_{i}$ is the fraction of the total demand $\alpha$ assigned to the boiler $i$, and evaluating their corresponding costs $J_{k_{1} k_{2}}$ through equation (6). The relative cost savings $D_{k_{1} k_{2}}$ are computed analogously to the two-boilers case:

$$
D_{k_{1} k_{2}}:=100 \frac{J_{k_{1} k_{2}}-J^{*}}{J^{*}},
$$




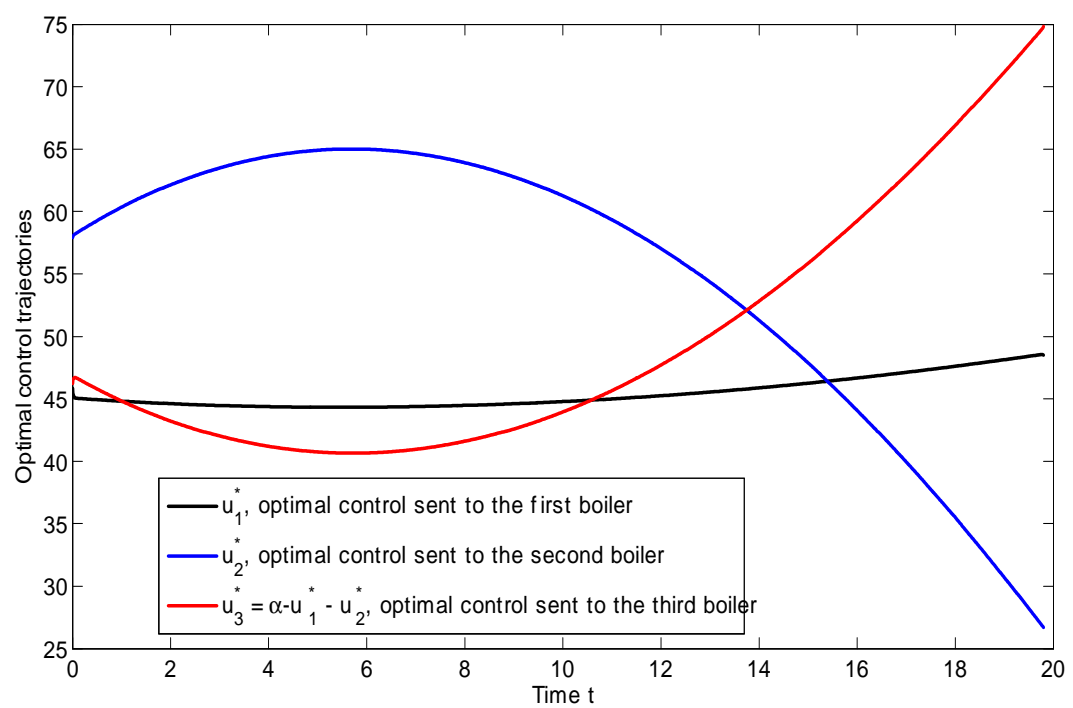

Figure 10: Optimal control for three boilers in parallel.

The numerical values are reported in Table 2 for different admissible combinations of $\left(u_{1}, u_{2}, u_{3}\right)$. The behavior of the savings according to variations in the participation of the members does not a seem to have a simple explanation, but again it is found that there exist at least a $10 \%$ of cost savings in using time-varying controls, as was the case in the two-boilers case study.

Comparison concerning energy losses. Although this paper was up to now devoted to quadratic costs, to the advantage that an explicit optimal solution was analytically worked out, previous results may be also useful for other optimization criteria to be assessed. Here we will test a new cost criterion $J^{B}$ that includes the trajectory energy loss defined by

$$
E_{\text {loss }}:=\sum_{i=1}^{3} E_{\text {fuel }, i}-\sum_{i=1}^{3} E_{\text {Vapor }, i}=\sum_{i=1}^{3} \int_{0}^{T}\left[x_{2 i}(t)-x_{1 i}(t)\right] d t,
$$

and also a quadratic final penalization as before. This cost $J^{B}$, for constant controls $u_{k_{1} k_{2}}$ takes then the form

$$
J_{k_{1} k_{2}}^{B}:=E_{l o s s, k_{1} k_{2}}+\left(x_{k_{1} k_{2}}(T)-\bar{x}\right)^{\prime} S\left(x_{k_{1} k_{2}}(T)-\bar{x}\right) .
$$

It must be noticed that, without a final penalization, the optimal solution for constant controls (in a finite horizon context) would trivially be the strategy that uses less fuel. A good enough approximation to the optimal combination 


\begin{tabular}{cccc}
\hline$k_{1}$ & $k_{2}$ & $k_{3}$ & $D_{k_{1} k_{2}}$ \\
\hline 0.2 & 0.3 & 0.5 & $36 \%$ \\
0.2 & 0.4 & 0.4 & $23 \%$ \\
0.2 & 0.5 & 0.3 & $47 \%$ \\
0.3 & 0.2 & 0.5 & $43 \%$ \\
0.4 & 0.2 & 0.4 & $34 \%$ \\
0.5 & 0.2 & 0.3 & $53 \%$ \\
0.2 & 0.3 & 0.2 & $38 \%$ \\
0.3 & 0.5 & 0.2 & $42 \%$ \\
0.4 & 0.4 & 0.2 & $20 \%$ \\
0.3 & 0.4 & 0.3 & $10 \%$ \\
\hline
\end{tabular}

Table 2: Optimal cost compared against the outcome of applying a constant set-up of different magnitude, where $k_{i}$ is the fraction of the total demand $\alpha$ for the boiler $i$, and $D$ shows the porcentage of cost savings giving by Eq. (49).

$k_{1} k_{2}$ can be obtained by direct calculation of the trajectories for the three boilers of this example, for a representative number of admissible pairs $\left(k_{1}, k_{2}\right)$. The $\operatorname{cost} J^{B}$ changes little with the parameter $S$, so it was tuned to make the optimal solution $u_{k_{1} k_{2}}^{o p t}$ for constant controls coincide with that one appearing in Table 2 , namely for $k_{1}=0.3, k_{2}=0.4, k_{3}=0.3$ (see Figure 11). Now the question is: how would our time-varying control $u^{*}$ of equation (22) perform under the new cost criterion of equation (51), both calculated for the same value of $S$ ? In numbers, this would amount to compare the optimal value $\left(J_{k_{1} k_{2}}^{B}\right)^{o p t}$ for the new cost, i.e.

$$
\left(J_{k_{1} k_{2}}^{B}\right)^{\text {opt }}:=\min _{k_{1}, k_{2}}\left\{J_{k_{1} k_{2}}^{B}, \text { all admissible }\left(k_{1}, k_{2}\right)\right\},
$$

against the formula in equation (51) applied to the optimal time-varying control $u^{*}$, i.e.

$$
J^{B}\left(u^{*}\right):=\sum_{i=1}^{3} \int_{0}^{T}\left[x_{u^{*}, 2 i}(t)-x_{u^{*}, 1 i}(t)\right] d t+\left(x_{u^{*}}(T)-\bar{x}\right)^{\prime} S\left(x_{u^{*}}(T)-\bar{x}\right) .
$$

The answer was in favor of the time-varying optimal control, precisely

$$
D_{B}:=100 \frac{\left(J_{k_{1} k_{2}}^{B}\right)^{o p t}-J^{B}\left(u^{*}\right)}{J^{B}\left(u^{*}\right)}=100 \frac{J_{0.3,0.4}^{B}-J^{B}\left(u^{*}\right)}{J^{B}\left(u^{*}\right)}=19 \% .
$$

Simulation test for identical boilers. In the hypothetical case that all $n$ members of the team have the same characteristics (reflected in the same state model and same evaluation parameters), the optimal allocation of demands could be predicted by a simple calculation, namely

$$
u_{i}^{*}(t) \equiv \frac{\alpha}{n}, i=1, \ldots, n-1 .
$$




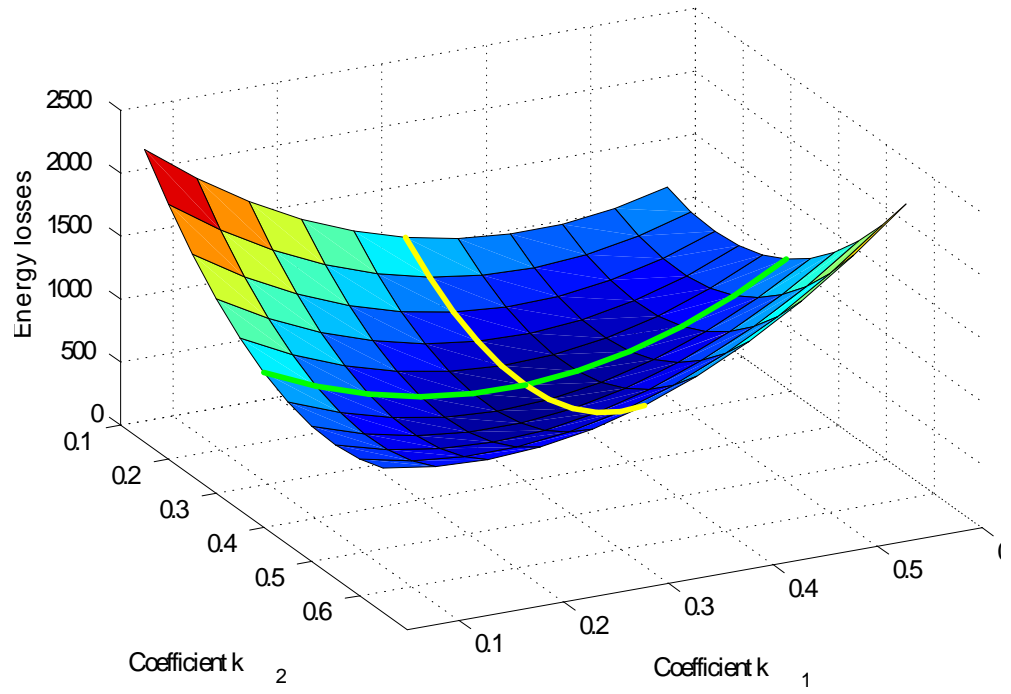

Figure 11: Evaluation of energy losses $J_{k_{1} k_{2}}^{B}$ with final penalizations $(S=55 I)$ as in equation (51), generated by different admissible constant controls $u_{k_{1} k_{2}}$ as defined in equation (48).

This is illustrated in Figure 12, for the numerical experiments with two an three identical boilers.

\section{Conclusions}

An optimal control strategy for dynamically changing the set-points for produced vapor to a group of $n$ boilers in parallel was proven and illustrated. The resulting feedback law minimizes the sum of two competing cost objectives: the departure of the production from the target, and the consumption of fuel during the optimization period. This provides online optimal allocation of demands to each of a number of boilers in multilayer controlled operation. The control has $n-1$ degrees of freedom, since all individual targets must sum up to the total demand signal coming from the supervisory control of the plant. This lack of freedom introduces a linear-affine structure for the dynamics of the problem, when posed for the whole group of boilers. The combination of quadratic individual costs for the boilers results in a quadratic total cost for the group. Then, the treatment for the linear-affine enlarged dynamics and quadratic cost differs from the usual LQR setup, resembling the equations associated with a tracking problem. The solution for the deterministic case is found in terms of the Riccati equation plus a feed through time-varying vector that can be stored once and for all, the changes in total demand are simply handled by introducing 


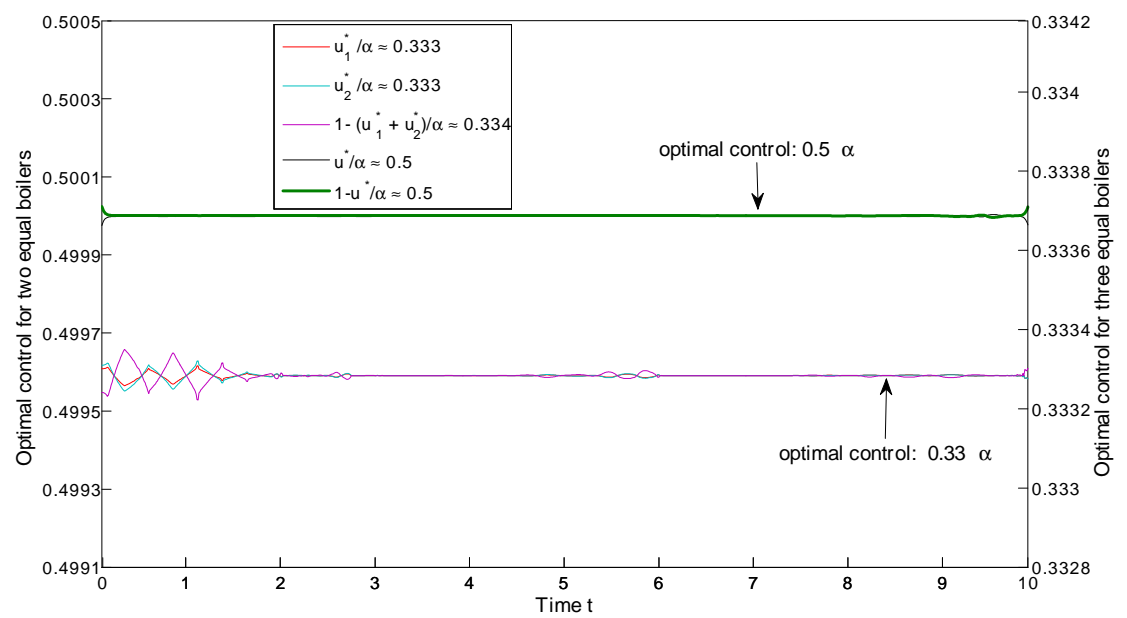

Figure 12: Numerical optimal controls calculated for two identical boilers (resulting in $u_{i}^{*} \cong \alpha / 2$ ); and for three identical boilers (resulting in $u_{i}^{*} \cong \alpha / 3$ ).

the new value as a factor in the feedback law. This feedback form of the control implies robustness with respect to state perturbations. Control parameters are calculated offline and do not need to be recalculated after changes on the total demand.

The choice of linear models for each boiler also allows to refine the deterministic result and obtain an optimal control from the stochastic viewpoint, coping with general disturbances like changes in fuel composition and noisy measurements. This is possible by a rigorous application of the Separation Principle and the addition of a Kalman filter.

In some situations, savings in cost have shown to be significant with respect to those generated by piecewise-constant strategies, above all when the optimal control indicates a departure from the equipartition of the total demand. In all cases there was a relative saving exceeding $10 \%$. Other optimization criteria deserve further investigation, although energy losses corresponding to our optimal control resulted smaller than those corresponding to piecewise-constant controls in a simulation for three boilers. In conclusion, the results presented here seem attractive for practical implementation in chemical plants.

\section{References}

[1] Saidur R, Ahamed JU, Masjuki HH. Energy, exergy and economic analysis of industrial boilers. Energy Policy 2010;38:2188-2197. 
[2] Liao Z, Dexter AL. The potential for energy saving in heating systems through improving boiler controls. Energy and Buildings 2004;36:261-271.

[3] Peeters L, Van der Veken J, Hens H, Helsen L, D'haesele W. Control of heating systems in residential buildings: Current practice. Energy and Buildings 2008;40:1446-1455.

[4] Moradi H, Bakhtiari-Nejad F. Improving boiler unit performance using an optimum robust minimum-order observer. Energy Conversion and Management 2011;52:1728-1740.

[5] Shide W, Jia L, Yuxin L. Energy Saving and Optimal Control of Gas Boiler Group. In: Power and Energy Engineering Conference 2009;27-31.

[6] Sniders A, Komass T. Invariant Method of Load Independent Pressure Control in Steam Boiler. Electrical, Control and Communication Engineering 2012;1: $5-10$.

[7] Krüger K, Franke R, Rode M. Optimization of boiler start-up using a nonlinear boiler model and hard constraints. Energy 2004;29:2239-2251.

[8] Liukkonen M, Hiltunen T. Adaptive monitoring of emissions in energy boilers using self organizing maps: An application to a biomass-fired CFB (circulating fluidized bed). Energy 2014;73:443-452.

[9] Bujak J. Optimal control of energy losses in multi-boiler steam systems. Energy 2009;34:1260-1270.

[10] Havlena V. Coordination in multilayer process control and optimization schemes. European Patent EP 1563347 B1, published September 30, 2009, Bulletin 2009/40.

[11] Hatzopoulos D, Koutsopoulos I, Koutitas G, Van Heddeghem W. Dynamic virtual machine allocation in cloud server facility systems with renewable energy sources. In: IEEE International Conference on Communications 2011;4217-4221.

[12] Xu J, Zeng Z. Applying Optimal Control Model to Dynamic Equipment Allocation Problem: Case Study of Concrete-Faced Rockfill Dam Construction Project. Journal of Construction and Engineering Management 2011;137(7):536-550.

[13] Likins MR, LaSpisa RJ. U.S. Patent Number 4,583,497, April 22; 1986.

[14] Härkegård O, Torkel Glad S. Resolving actuator redundancy-optimal control vs. control allocation. Automatica 2005;41 $137-144$.

[15] Costanza V, Rivadeneira PS. Online suboptimal control of linearized models. Systems Science 83 Control Engineering 2014;2:379-388. 
[16] Sontag ED. Mathematical Control Theory. 2nd ed. New York: Springer; 1998.

[17] Costanza V, Rivadeneira PS. Enfoque Hamiltoniano al control óptimo de sistemas dinámicos. Saarbrücken: OmniScriptum; 2013.

[18] Costanza V. Parametric uncertainty and disturbance attenuation in the suboptimal control of a nonlinear electrochemical process. Optimal Control Applications \& Methods 2007;28(3): 209-228.

[19] Fleming WH, Rishel RW. Deterministic and Stochastic Optimal Control. Springer, New York; 1975.

[20] Faurre P. Teoría de Control - Filtrado Estadístico e Introducción al Control Estocástico. Cuadernos del Instituto de Matemática Beppo Levi $\mathrm{N}^{\circ} 5$, Universidad Nacional de Rosario, Rosario, Argentina; 1973.

[21] Bryson A Jr, Ho Y. Applied Optimal Control. Revised printing. New York: John Wiley and Sons;1975.

[22] Das S, Pan I, Halder K, Das S, Gupta A. Optimum weight selection based $\mathrm{LQR}$ formulation for the design of fractional order $\mathrm{PI}^{\lambda} \mathrm{D}^{\mu}$ controllers to handle a class of fractional order systems. IEEE International Conference on Computer Communication and Informatics 2013;1-6.

[23] Robinson JD. A linear quadratic regulator weight selection algorithm for robust pole assignment. Air Force Institute of Technology, USA; 1990. 